\title{
CONNECTION BETWEEN OPTICAL AND HIGH FREQUENCY RADIO VARIABILITY IN AGN
}

\author{
M. TORNIKOSKI and E. VALTAOJA \\ Metsähovi Radio Research Station, SF-02540 Kylmälä, Finland \\ and \\ A.G. SMITH and A.D. NAIR \\ Astronomy Dept., University of Florida, Gainesville, Fl-32611, USA
}

We have been searching for correlated optical and radio variability in large temporal data sets of 22 extragalactic radio sources. The optical data were obtained with the 76-cm reflector at the Rosemary Hill Observatory in Florida, USA. The radio data were obtained at two different sites: 22,37 and some of the $90 \mathrm{GHz}$ data at the Metsähovi Radio Research Station, Finland, and 90 and $230 \mathrm{GHz}$ data at the Swedish-ESO Submillimetre Telescope (SEST) on La Silla, Chile. Because the SEST data unfortunately reaches only back to 1988 , the 90 and $230 \mathrm{GHz}$ data were complemented by the IRAM data from Steppe et al. (A\&AS 75, 1988 and A\&AS 96, 1992).

For the correlation analysis we used the discrete correlation function method described in Edelson \& Krolik (ApJ 341, 1988), suitable for unevenly sampled data sets. Even though the data streams covered 10 years or more, we ran the analysis collecting only information of the correlations with time lags of less than about 1000 days. We also inspected the optical and radio flux curves visually both for discrete events and long-term behaviour.

We found radio-optical connections in a significant fraction of our sample. In several cases optical flares were found to occur simultaneously with the high frequency radio flares. The radio-optical connection was very clear in 10 cases, in 6 of which the time delays were practically zero, i.e. less than our bin size of 30-50 days. In 5 of the remaining sources at least some optical flares seemed to be connected with radio flares, often the only notable events in the radio data sets. All of the sources in which strong radio-optical correlation was found were either highly polarized quasars or BL Lac objects, and they were all among the most frequently observed sources.

It is possible that there are several types of optical events, some of which are not directly connected to radio events. We suggest however that the converse may be true, with optical flares occuring synchronously with all the high radio frequency flares and with time delays of zero to several hundred days at lower radio frequencies. Since optical flares are typically very rapid, it is easy to miss many such events due to undersampling. We believe that strong radio-optical connections would be detected in many more sources if the sampling density were high enough to ensure detecting all the notable flares at both optical and high radio frequencies.

411

T. J.-L. Courvoisier and A. Blecha: Multi-Wavelength Continuum Emission of AGN, 411.

(C) 1994 IAU. Printed in the Netherlands. 